\title{
Fundamental Diagram Estimation through Passing Rate Measurements in Congestion
}

\author{
Nicolas Chiabaut*, Christine Buisson, \\ Ludovic Leclercq
}

\begin{abstract}
Classically, fundamental diagrams are estimated from aggregated data at a specific location. Such a measurement method may lead to inconsistency which mainly explain the current controversy about their shape.

This paper proposes a new estimation method based on passing rate measurements along moving observer paths. Under specific assumptions, it can be proved that in congestion passing rate is independent of the traffic flow states.

This property allows (i) to prove that a linear fundamental diagram is suitable to represent traffic flow behavior involved in the Next Generation Simulation Model (NGSim) dataset; (ii) to fit its two parameters: the congested wave speed and the jam density.
\end{abstract}

Index Terms-Passing rate, macroscopic, NGSIM, fundamental diagram, equilibrium traffic state, traffic flow theory.

\section{INTRODUCTION}

$\mathbf{T}$ HE fundamental diagram (FD), i.e. the relationship between flow $q$ and density $k$, encompasses both equilibrium traffic states definition [6] and traffic wave structure, i.e. how two separate equilibrium states propagate [14], [19]. Data used to estimate this relationship are usually obtained from loop detectors at a specific location and are aggregated over time intervals ranging from 30 second to 1 minute [1], [7], [9], [12]. The aggregation can trigger three potential drawbacks: (i) various traffic states are mixed in measurements, (ii) data corresponding to equilibrium states are difficult to identify, (iii) the wave structure cannot be captured at a single location. These points are explained hereafter.

(i) Free flow and congested traffic states might be averaged over arbitrary time intervals. Thus, different or transient states are mixed into a unique value $(k, q)$. Moreover, data from several lanes are often gathered without checking that all lanes behave similarly [11]. Even single lane data can be corrupted by lane changing effects. Mixing different traffic states may explain the various shapes of FDs, $Q(k)$, reported by a lot of papers since the early 80's [7], [8], [10].

(ii) At a specific location, equilibrium and transient phases are difficult to distinguish. Cassidy proposed in [1] an empirical method to determine nearly-stationary situations from

*Corresponding author

The authors belong to the Université de Lyon, INRETS / ENTPE, LICIT, Rue Maurice Audin, F-69518 Vaulx En Velin CEDEX, France (e-mail: nicolas.chiabaut@entpe.fr; christine.buisson@entpe.fr; ludovic.leclercq@entpe.fr) observed cumulative curves. However, this method is inefficient to detect these nearly-stationary situations in the vicinity of shockwaves. Thus, a high frequency of transitions prevents equilibrium states to be identified with this method. Furthermore, the inspection of the cumulative curves is complicated to implement.

(iii) In traffic flow models, the FD defines how traffic states propagate. It is then appealing to base FD estimation on spatial measurements. Note that such an approach is consistent with EDIE's definition of equilibrium [5]. To the authors' knowledge, only cross correlation technique on cumulative curves at two successive locations [2], [20] fall in this category. These studies estimated that the maximum velocity for backward moving waves range from $15 \mathrm{~km} / \mathrm{h}$ to $20 \mathrm{~km} / \mathrm{h}$. However, this method can only determine wave speeds in congestion and does not reflect what happens between the measurement locations.

This paper proposes a new method to estimate the fundamental diagram (FD) in congestion. This method is based on theoretical properties of passing rates, i.e. flows that cross a moving observer. It overcomes the three abovementioned drawbacks. (i) Individual trajectories coming from the NGSim dataset [17] are used. Such data permit lane by lane analysis ruling out effects of lane-changing. (ii) Relevant paths for the moving observer, only run into equilibrium states. (iii) Passing rate measurements are continuous in time and in space by definition. Furthermore, the proposed method will reinforce the assumption that the FD is linear in congestion. Consequently, only two parameters, the congested wave speed $w$ and the jam density $k_{x}$, have to be estimated.

This method attempts to determine lane specific FD in congestion for the NGSim experimental site. Towards this end, section 2 identifies congested stable platoons in traffic flow. Section 3 formulates the framework of the proposed method and section 4 presents the results for on-field data.

\section{NGSIM DATASET ON THE HIGHWAY I-80}

In this paper, data are coming from the American project Next Generation Simulation (NGSim). Vehicle trajectories of the I-80 freeway near Berkeley, California, have been selected to support the study. Fig. 1 provides a sketch of the experimental site.

Video recording of vehicle trajectories was performed during 5 hours, on April 13, 2005. Ten frames per second were 
(a)

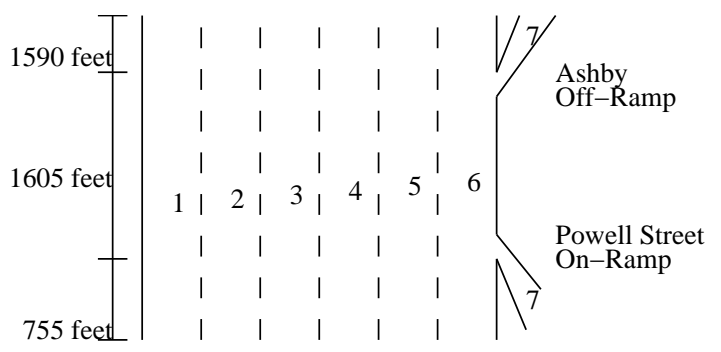

(b)

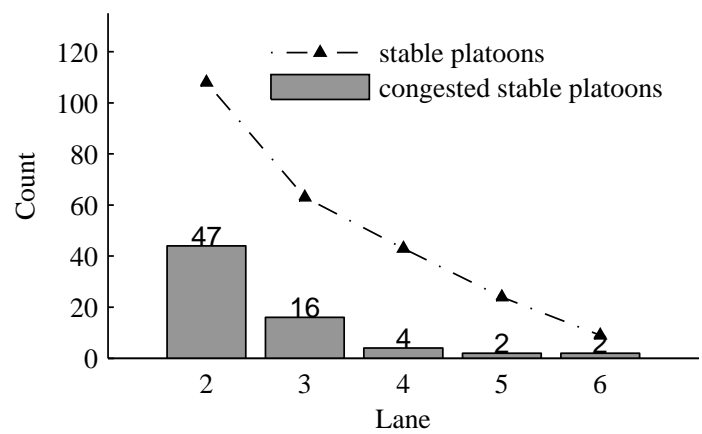

Fig. 1. NGSim Dataset. (a) Site description (b) Total number of stable platoons, total number of congested stable platoons for each lane

available. A sample of 45 minutes was processed and used for this study.

To estimate the FD on the experimental site, one should focus on stable platoons. A bunch of vehicles is said to form a stable platoon when more than 4 vehicles stay in the same lane without any overtaking process. This condition ensures that only stationary situations are considered. Thus, 244 stable platoons can be identified in the dataset on lanes 2 to 6 .

This paper focuses on congested states. Then, a subset $S_{1}$ is created among stable platoons where the leader speed is below $45 \mathrm{~km} / \mathrm{h}$. Note that lane 1 is an HOV lane which is less congested. We decided to exclude lane 1 from our study. Thus, $S_{1}$ contains 71 congested stable platoons. Notice that the distribution of the platoons is strongly correlated to the lane number. Lane-changing are frequent on lanes in the vicinity of the weaving section (lane 6). Consequently, these lanes (4, 5 and 6) do not present enough congested stable platoons to estimate the FD accurately. They have been excluded from our dataset. The final sample only contains 368 vehicles which form 63 congested stable platoons on lane 2 and 3 (Fig. 1). Such a size is too low to estimate a FD with classical methods found in literature but will be sufficient for the proposed new method.

\section{ESTIMATING THE FUNDAMENTAL RELATIONSHIP BY PASSING RATES MEASUREMENTS}

This section shows that evaluating discrepancy in passing rate measurements for various moving observer speeds can provide an accurate method to estimate FD.

\section{A. Definition and properties of the passing rate}

We consider the trajectory $\mathscr{C}$ from point $A$ to point $B$ (Fig. 2) of an observer moving backwards at constant speed $v>0$. Therefore, the associated passing rate at which vehicles cross the trajectory $\mathscr{C}$ is:

$$
r\left(\mathscr{C}_{A \rightarrow B}\right)=\frac{\Delta n}{\Delta t}
$$

where $\Delta n$ is the number of vehicles that pass the observer and $\Delta t$ is the time required by the observer to reach $B$ from $A$ (see Fig. 2).

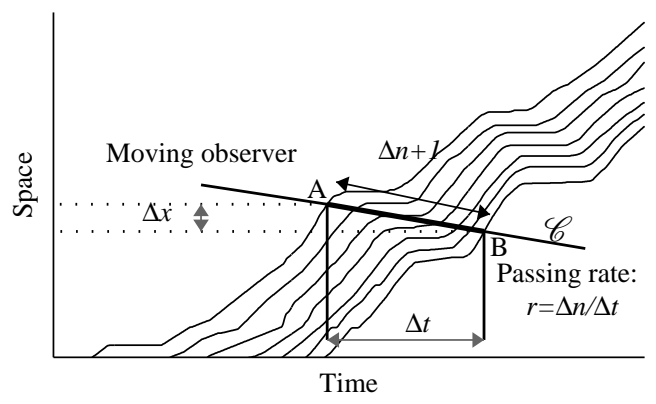

Fig. 2. Passing rate of a moving observer of trajectory $\mathscr{C}$

Let $Q$ denote the FD that holds in the considered $t-x$ plane. Within the framework of kinematic wave models, if traffic conditions are stationary along the path $\mathscr{C}$, the density is constant and equal to $k$ and the flow is equal to $q=Q(k)$. Note that the definition of $k$ supposes that the traffic flow is continuously approximated. For more details see [4].

Consequently, the passing rate on $\mathscr{C}$ can also be expressed as in [15]:

$$
r\left(\mathscr{C}_{A \rightarrow B}\right)=Q(k)+k v .
$$

The above-mentioned passing rate formulation is independent of the FD shape. However, provided that $Q(k)$ is linear in congestion and that the traffic is congested, equation (2) yields to [15]:

$$
r\left(\mathscr{C}_{A \rightarrow B}\right)=w k_{x}-k(w-v) .
$$

where $w$ (wave speed) and $k_{x}$ (maximal density) are the two parameters of $Q$.

Furthermore, the passing rate can also be expressed with respect to the platoon leader speed $u$. Indeed, the speed $u$ is equal to the flow speed $q / k$ in this case [16].

$$
r(u, v)=w k_{x}-\frac{w k_{x}}{w+u}(w-v) .
$$

It appears that, when the observer travels at speed $v$ equal to $w$, the passing rate is constant and equal to $w k_{x}$ independently of $u$. To illustrate this property, Fig. 3 shows a theoretical platoon of 5 vehicles where the leader changes its speed from $u_{1}$ to $u_{2}$. On the left side (Fig. 3a), the observer moves at speed $w$. Thus the time required to reach the last vehicle is the same in the two regions $\left(u_{1}\right.$ and $\left.u_{2}\right)$. Passing rate is then 
(a)

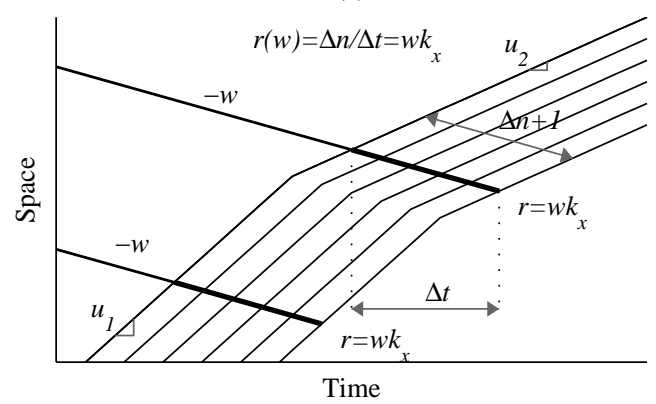

(b)

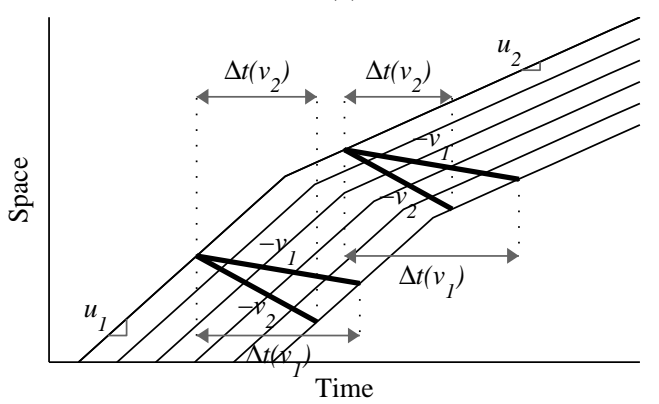

Fig. 3. Discrete calculus of the passing rate for an observer moving at speed (i) $v=w$ and at speed (ii) $v \neq w$

uniform along the trajectory and equal to $w k_{x}$ regardless of the leader speed. On the right side (Fig. 3b), the observer moves at a speed $v \neq w$. Then time required to reach the last vehicle is no longer the same for the two states $u_{1}$ and $u_{2}$. In this case, time period $\Delta t\left(v_{1}\right)$ increases with the flow speed whereas $\Delta t\left(v_{2}\right)$ decreases with $u$.

It is worth noting that the reciprocal statement of (2) is also true. Indeed, if the passing rate along the trajectory of an observer moving at $v$ is constant and equal to $C$ regardless of vehicles speed, then equation (2) reduces to:

$$
Q(k)=C-k v,
$$

Consequently, the FD is linear in congestion.

This yields to the following theorem. A FD is linear in congestion, if and only if, it exists a moving observer speed $v^{*}$ thereby the associated passing rate is uniform among the observed traffic states. Furthermore $v^{*}$ is then equal to $w$.

The paper will now propose a method to determine $v^{*}$ on on-field data. Passing rate measurements, $r(u, v)$ will be computed along the selected platoons trajectories with respects to $u$ and $v$. For any point of the leader trajectory, one can determine the time period $\Delta t$ required by the observer to reach the last trajectory (Fig. 3). Then $r(u, v)$ is calculated as $\Delta n / \Delta t$ where $(\Delta n+1)$ is the number of vehicles involved in the platoon. Let $[r(u, v)]$ denote the dataset of passing rate measurements obtained along the leader trajectory for $v$, $[r(u, v)]=\{r(u, v), \forall u$ observed $\}$. Then, various values of $v$ will be tested. Finally, $v^{*}$ will be equal to the $v$-value providing the less discrepancy in $[r(u, v)]$.

\section{B. Passing rate measurements for various observer speeds}

It is appealing to formally assess the variations in $[r(u, v)]$. To this end, one should study how the passing rate vary along the platoon's trajectory. For any speed $v \neq w, r(u, v)$ is modified when $u$ changes. If the leader decelerates from speed $u_{1}$ (state 1 on the diagram) to speed $u_{2}$ (state 2 on the diagram), the absolute change in passing rate measurements is equal to:

$$
I(v)=\frac{w k_{x}}{\left(w+u_{1}\right)\left(w+u_{2}\right)}\left|u_{1}-u_{2}\right||v-w| .
$$

Value of $I$ depends on $v$ but also on the leader's speed variation, $\left|u_{1}-u_{2}\right|$. As an illustration, Fig. 4 shows $I$ for two different observer speeds $v_{1}$ and $v_{2}$ in the case of a leader reducing its velocity from $u_{1}$ to $u_{2}$. For any point $i$, one can draw a line of slope $-v$. This line intercepts the $q$-axis at the level of $r\left(u_{i}, v\right)$. Notice that $I$ is null when $v=w$ regardless of $u$.

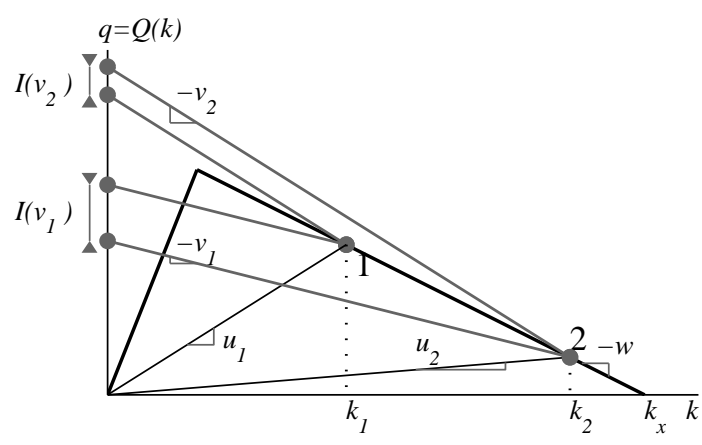

Fig. 4. Change in passing rate measurements for two observer's speeds $v_{1}$ and $v_{2}$ when the leader decelerates from $u_{1}$ to $u_{2}$

Evolution of $I$ with respect to $v$ can be drawn for various values of $\left|u_{1}-u_{2}\right|$ (Fig. 5). It appears that the greater the leader speed variation, the sharper the objective function (relative standard deviation of passing rate values). This sharp shape prevents from drawbacks of existing methods like flat objective functions that may lead to multiple optimal parameters [18].

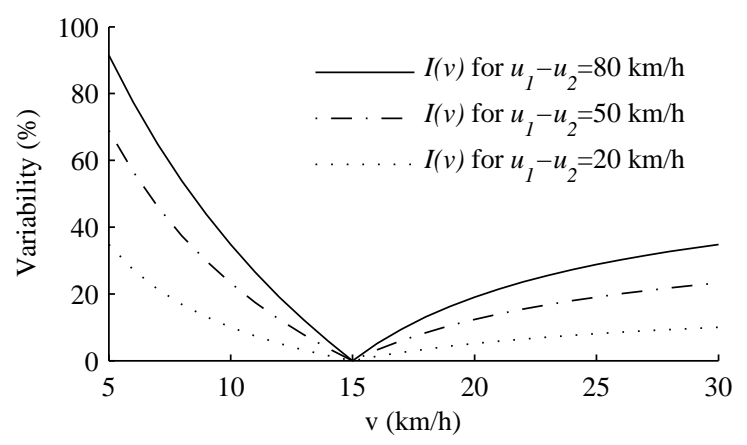

Fig. 5. Evolution of the change in passing rate measurements for leader speed variation of $80 \mathrm{~km} / \mathrm{h}, 50 \mathrm{~km} / \mathrm{h}$ and $20 \mathrm{~km} / \mathrm{h}$

If $u$ is uniform along the test bed, passing rate remains constant regardless of $v$. Hence, changes in $u$, i.e. shockwaves, 
have a major influence on variation of $r(u, v)$. They generate discrepancies in passing rate measurements when $v \neq w$. Thus, to magnify the effect of shockwaves, mean values of $r(u, w)$ by range of speed centered in $\bar{u}$ have been calculated. We will denote these mean values $R(\bar{u}, w)$.

Note that the averaging process has also the asset to smooth the errors due to the data. Indeed, let us consider a uniform measurement error $\varepsilon$ of about $10 \%$ between the recorded value $r$ and the real value $\hat{r}$ of the passing rate:

$$
r(u, v)=\hat{r}(u, v) \pm \varepsilon \approx \hat{r} \pm 10 \%,
$$

Now, mean values of $r$ are calculated by range of $u$. To guaranty that each subset contains enough measurements, the width of the range is fixed at $5 \mathrm{~km} / \mathrm{h}$. Thus, more than $n>=$ 200 values of $r(u, v)$ have been recorded by speed interval for any value of $v$. According to the Central Limit Theorem the estimated mean point is:

$$
R(\bar{u}, v)=\mu \pm 1.96 \frac{\sigma}{\sqrt{n}} \approx \mu \pm 2.8 \%,
$$

where $\mu$ is the real value of the passing rate for the mean range speed $R(\bar{u}, v)$ and $\sigma$ is the standard deviation of $\varepsilon$.

Thereby doing, averaging passing rate values by range of speed reduces the dispersion caused by the measurements error in dataset.

Finally, discrepancies in passing rate measurements will be evaluated by the relative standard deviation $s$ of $[R(\bar{u}, v)]=$ $\{R(\bar{u}, v), \forall \bar{u}$ observed $\}$. The wave speed $w$ corresponds to $v^{*}$ such as:

$$
v^{*}=\arg \left[\min _{v}[s([R(\bar{u}, v)])]\right] .
$$

\section{Verifying the theory on simulated data}

The proposed method relies on the assumption that the FD holds on stable platoons. This is equivalent to assume that vehicles inside the platoon follows as specific $\mathrm{CF}$ rule: Newell's CF model [16], [3], [13]. To check that the proposed method does not introduce a bias on data that perfectly match this assumption, we are going to test it on simulated congested trajectories generated with Newell's CF model.

For a platoon formed of $n+1$ vehicles, parameters of the FD are randomly selected. Trajectories are then computed according to the values of $w$ and $k_{x}$. Note that the leader of the platoon changes its speed at least one time to induce shockwaves. Passing rate are measured for any leader speed as in Fig. 3. Value of $v$ that minimizes $s([R(u, v)])$ can be determined.

Fig. 6 illustrates this process when $w=15 \mathrm{~km} / \mathrm{h}$ and $k_{x}=150 \mathrm{veh} / \mathrm{km}$. The method determines $v^{*}$ as equal to the initial value of $w$. Evolution of $s([R(u, v)])$ with respect to $v$ is depicted in Fig. 6. It confirms that the shape of the objective function is very sharp. Note that $v^{*}$ corresponds to a criterion equal to $0 \%$.

The study was performed for various platoons sizes and values of $w$ and $k_{x}$. In any case, $v^{*}$ is equal to $w$ and leads to a null relative standard deviation. This is not surprising since the proposed method aims to determine the shift leading to the best fit between the leader's trajectory and the last trajectory.
This exactly corresponds to Newell's model where vehicles trajectories in congestion are calculated by shifting the leader's trajectory in time and space [16]. Consequently, the proposed method does not introduce any bias.

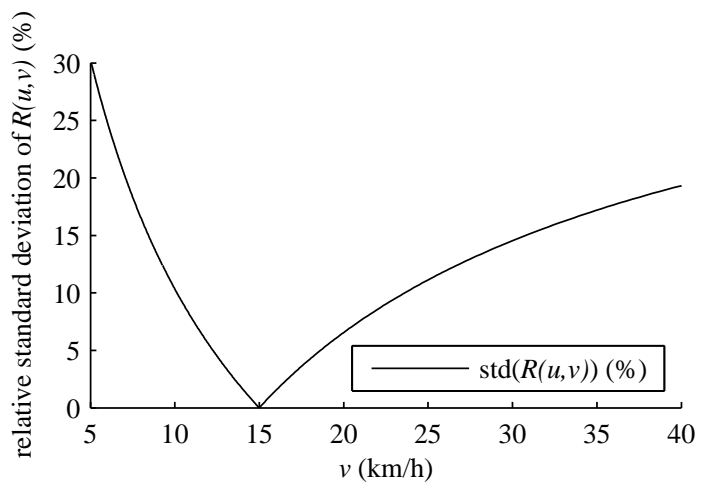

Fig. 6. Evolution of the criterion versus the observer speed when $w=15$ $\mathrm{km} / \mathrm{h}$

\section{ESTIMATION OF THE CONGESTED PART OF THE DIAGRAM AND ANALYSIS OF THE RESULTS}

In this section the method is performed on platoon trajectories selected in section II. Then, measurement are gathered to estimate lane specific FD.

\section{A. Filtering and measurements}

Passing rate measurements are performed along each platoon leader trajectories of the dataset every 1 second (Fig. 7). A value of 1 second is a reasonable value to ensure a sufficient number of measurements but also a fast computing time. Then, $r(u, v)$ are calculated along the leader trajectory. We obtain at least 3795 measurements in $[r(u, v)]$ on lanes 2 and 3 for each tested $v$-value.

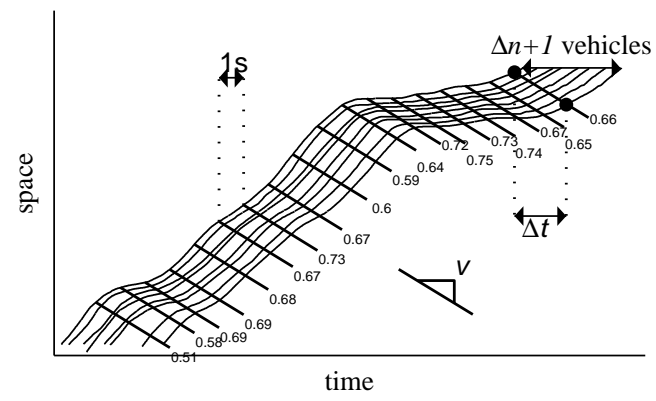

Fig. 7. Measurement process of passing rates

These measurements are then averaged by range of $u$ leading to $[R(\bar{u}, v)]$. Ranges of 5 kilometers seem to be a good compromise to ensure a sufficient number of values in $[R(\bar{u}, v)]$. The maximal leader speed is close to $45 \mathrm{~km} / \mathrm{h}$. Thus, 9 mean values of $R(\bar{u}, v)$ are calculated for each lane. Then, the relative standard deviation of these measurements is calculated:

$$
s(v)=\frac{\sum_{i=1}^{9}\left(R\left(\bar{u}_{i}, v\right)-\overline{R(\bar{u}, v)}\right)^{2}}{\overline{R(\bar{u}, v)}}
$$


(a)

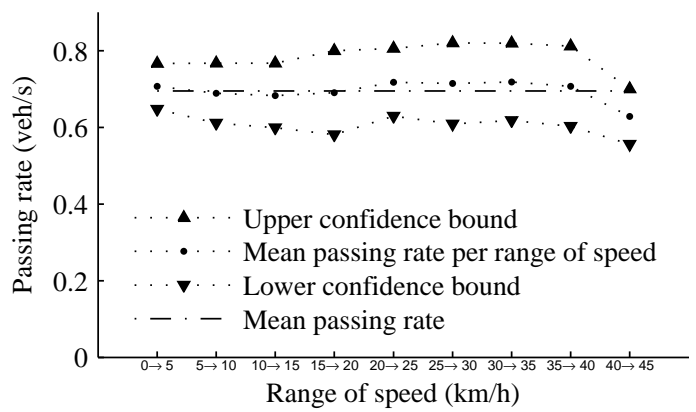

(b)

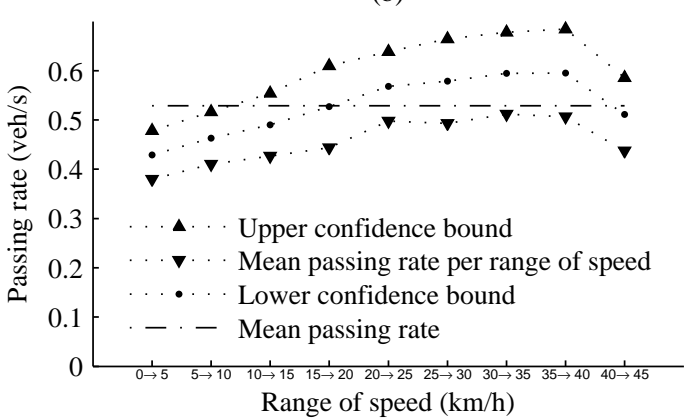

Fig. 8. (a) Confidence intervals on lane 2 for an optimal $v=18.9 \mathrm{~km} / \mathrm{h}$ (b) Confidence intervals on lane 2 for a non optimal $v=15 \mathrm{~km} / \mathrm{h}$

where $R\left(\bar{u}_{i}, v\right)$ is the mean passing rate for a leader speed comprised into $(i-1) * 5 \mathrm{~km} / \mathrm{h}$ and $i * 5 \mathrm{~km} / \mathrm{h}$, and $\overline{R(\bar{u}, v)}$ is the mean passing rate for the observed leader speed set.

The process is performed for $v$ varying in $[5 \mathrm{~km} / \mathrm{h} ; 20 \mathrm{~km} / \mathrm{h}]$ with a step of $0.1 \mathrm{~km} / \mathrm{h}$. Finally $v^{*}$ is equal to the value of $v$ which minimizes $s(v)$.

\section{B. Results}

The FD has been estimated separately for lane 2 and 3 . Interestingly, we cannot conclude to a common wave speed for both lanes.

1) Lane number 2: The previous method is illustrated by a detailed analysis of the results on lane 2. First of all, the number of measurements are not strongly dependent on the speed $v$ of the moving observer. Indeed, for every value of $v$ tested in the set $[5 \mathrm{~km} / \mathrm{h} ; 20 \mathrm{~km} / \mathrm{h}]$, more than 2500 measurements have been obtained. It ensures a sufficient sample to analyze the global behavior of traffic flow.

Fig. 8a depicts the mean values of the passing rate by range of leader speed for $v^{*}$. Fig. 8a presents also the confidence intervals (with a certainty of $\alpha=0.95$ ) of $R(\bar{u}, v)$. The difference among values of $R(\bar{u}, v)$ are not statistically significant. A common value can be assessed. On the contrary, Fig. $8 \mathrm{~b}$ shows that for a non-optimal speed $v, R(\bar{u}, v)$ cannot be considered as uniform among leader speed in congestion. Note that the associated value of $s\left(v^{*}\right)$ is equal to $3.5 \%$ which is very small. Thus, Newell's model accurately represents observed traffic flow dynamics at a macroscopic scale.

We can thus conclude that the FD is linear in congestion. The wave speed $w$ is equal to $v=18.9 \mathrm{~km} / \mathrm{h}$. Furthermore, the jam density can be assessed from the mean value of the passing rate. Indeed, $w \cdot k_{x}=2460 \mathrm{veh} / \mathrm{h}$, then $k_{x}$ is equal to $130 \mathrm{veh} / \mathrm{km}$. The congested part of the FD is completely estimated.

2) Lane number 3: The same method has been followed for lane 3 . With only 1225 measurements, we can conclude that the FD is also linear in congestion with $w$ equal to $14.5 \mathrm{~km} / \mathrm{h}$. Fig. 9 shows the confidence interval analysis and confirms that passing rate values are not different from on range of speed to another. The value of $s\left(v^{*}\right)$ is equal to $8.7 \%$. As previously, the jam density $k_{x}$ can be estimated from passing rate value and assessed at $138 \mathrm{veh} / \mathrm{km}$.

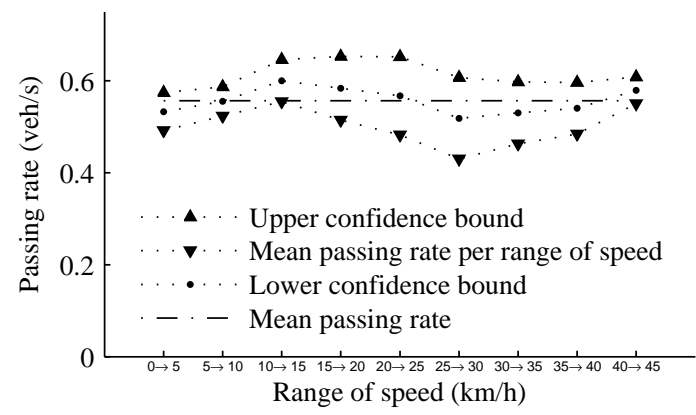

Fig. 9. Confidence intervals on lane 3 for the optimal value of $v$

Fig. 10 depicts the estimated congested part of the FD for lane 2 and lane 3 using the values of $w$ and $k_{x}$ previously found. Numbers of measurements are reminded. The confidence intervals of the passing rate (with a level of certainty $\alpha=0.95$ ) have been calculated. With respectively a confidence interval of $58 \mathrm{veh} / \mathrm{h}$ for lane 2 and $122 \mathrm{veh} / \mathrm{h}$ for lane 3 , it turns out that the lanes are statistically different. The closeness of an HOV lane (lane 1) may induce the variation of $w$ from lane 2 to lane 3. Further investigation on an additional dataset are required to corroborate or disprove this result.

Finally, the free flow part of the relationship has to be determined to obtain the whole diagram. This has not been carried out here but accurate methods can be found in literature (see for example [1]).

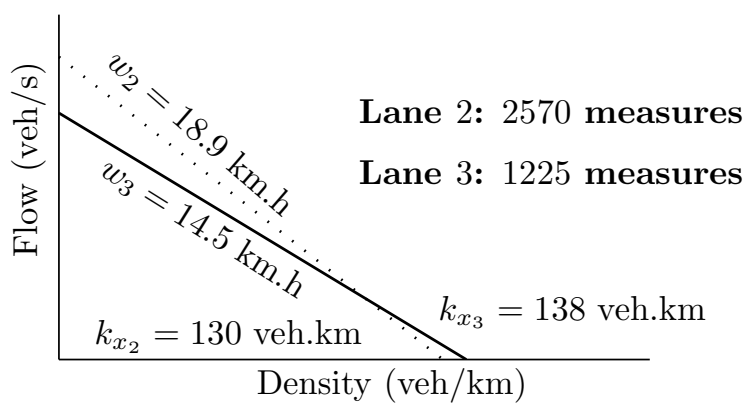

Fig. 10. Congested part of the fundamental diagram for lanes 2 and 3

\section{CONCLUSION}

This paper has introduced a new method to determine the congested part of the fundamental diagram. This method 
overcomes the main lacks of existing estimation processes: (i) a lane by lane analysis ruling out lane changing effects has been carried out; (ii) by measuring passing rate along a moving observer path, whose slope corresponds to wave speed, the same traffic state is encountered. Thus, no specific procedure is needed to identify equilibrium data. (iii) Passing rates are intrinsically suitable for capturing traffic wave effects.

The main insight of this paper is that the FD can be considered as linear in congestion. This has been experimentally proved from passing rate properties. Furthermore, the proposed method requires fewer observations than classical ones to estimate $w$ and $k_{x}$. Moreover, the findings seem to shed light on lane specific diagram with different values of $w$. This points out the importance of a lane by lane data analysis.

It should be noted that the process is free of error and is simple to implement if trajectories data are available. However, such data may be difficult and costly to collect. The authors are investigating how loop detector data from successive locations may permit to estimate passing rates. The proposed method could then be generalized to commonly available data.

\section{REFERENCES}

[1] M. J. Cassidy, "Bivariate relations in nearly stationary highway traffic," Transportation Research Part B: Methodological, vol. 32, no. 1, pp. 49-59, Jan. 1998.

[2] B. A. Coifman and Y. Wang, "Average velocity of waves propagating through congested freeway traffic," in 16th International Symposium on Transportation and Traffic Theory, H. S. Mahmassani, Ed. Mariyland, USA: Elsevier, 2005, pp. 165-179.

[3] C. F. Daganzo, "In traffic flow, cellular automata = kinematic waves," Transportation Research Part B: Methodological, vol. 40, no. 5, pp. 396-403, Jun. 2006.

[4] S. Darbha, K. Rajagopal, and V. Tyagi, "A review of mathematical models for the flow of traffic and some recent results," Nonlinear Analysis, vol. 69, pp. 950-970, 2008.

[5] L. C. Edie, "Car following and steady state theory for non congested traffic," Operations Research, vol. 9, pp. 66-76, 1961.

[6] B. D. Greenshields, "A study of traffic capacity," Proceedings of the Highway Research Board, vol. 14, pp. 448-477, 1935.

[7] F. L. Hall, Traffic flow theory: A state of the art report - revised monograph on traffic flow theory. Washington, D.C., USA: US Federal Highway Administration, 1996, ch. Traffic stream characteristics.

[8] F. L. Hall and L. M. Hall, "Capacity and speed-flow analysis af the queen elisaberth way in ontario," Transportation Research Record, vol. 1287, pp. 108-118, 1990 .

[9] K. Kockelman, "Changes in flow-density relationship due to environmental, vehicle, and driver characteristics," Transportation Research Record, vol. 1644, no. -1, pp. 47-56, Jan. 1998.

[10] M. Koshi, M. Iwasaki, and I. Okhura, "Some findings and an overview on vehicular flow characteristics," in Proceedings of the 8th International Symposium on Transportation and Traffic Flow Theory, V. F. Hurdle, E. Hauer, and G. Steuart, Eds. Canada: University of Toronto Press, 1983, pp. 403-426.

[11] J. A. Laval, "Linking synchronized flow and kinematic waves," in Traffic and Granular Flow '05, R. Khne, T. Poeschl, A. Schadschneider, M. Schreckenberg, and D. Wolf, Eds. Springer, 2005.

[12] L. Leclercq, "Calibration of flow-density relationships on urban streets," Transportation Research Record, vol. 1934, no. -1, pp. 226-234, Jan. 2005.

[13] — - "Hybrid approaches to the solutions of the "lighthill-whithamrichards" model," Transportation Research Part B: Methodological, vol. 41, no. 7, pp. 701-709, Aug. 2007.

[14] M. J. Lighthill and G. B. Whitham, "On kinematic waves ii. a theory of traffic flow on long crowded roads," Proceedings of the Royal Society A, vol. 229 , pp. $317-345,1955$.

[15] G. F. Newell, "A moving bottleneck," Transportation Research Part B: Methodological, vol. 32, no. 8, pp. 531-537, Nov. 1998.

[16] _ "A simplified car-following theory: a lower order model," Transportation Research Part B: Methodological, vol. 36, no. 3, pp. 195-205, Mar. 2002.
[17] NGSIM, "Next generation simulation," 2006. [Online]. Available: URL: http://ngsim.fhwa.dot.gov/

[18] S. Ossen and S. P. Hoogendoorn, "Validity of trajectory-based calibration approach of car-following models in the presence of measurement errors," in $88^{\text {th }}$ annual meeting of Transportation Research Board, 2008.

[19] P. I. Richards, "Shockwaves on the highway," Operations research, vol. 4, pp. 42-51, 1956.

[20] J. R. Windover and M. J. Cassidy, "Some observed details of freeway traffic evolution," Transportation Research Part A: Policy and Practice, vol. 35, no. 10, pp. 881-894, Dec. 2001.

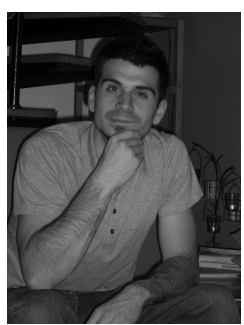

Nicolas Chiabaut received the M.Sc. degrees in civil engineering from the École Nationale des Travaux Publics de l'État (ENTPE) which belongs to the University of Lyon. He is currently finishing its $\mathrm{Ph} . \mathrm{D}$. degree with the LICIT, Laboratoire Ingenierie Circulation Transport (ENTPE / INRETS). His research is mainly focused on traffic flow theory. He has been recognized with the best paper award of the Young Researchers Seminar 2007 (ECTRI / FEHRL / FERSI) in Brno, Czech Republic.

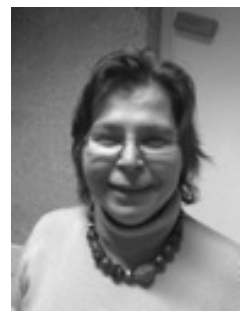

Christine Buisson received her $\mathrm{PhD}$ degree in 1991 as a physicist. Since fifteen years, she is full time researcher in traffic flow theory with INRETS. She is also responsible of the traffic modeling and transportation engineering courses at the École Nationale des Travaux Publics de l'État (ENTPE).

Her main interests are related to traffic flow modeling and congestion measurement and analysis.

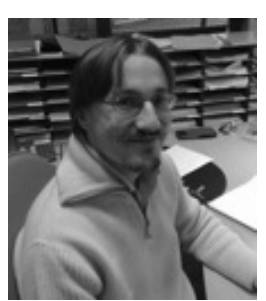

Ludovic Leclercq received the M.Sc degree and the $\mathrm{PhD}$ degree in civil engineering from Institut $\mathrm{Na}$ tional des Sciences Appliques (INSA-Lyon, France). $\mathrm{He}$ is currently a professor with the École Nationale des Travaux Publics de l'État (ENTPE) which belongs to the University of Lyon. He is a member of LICIT, Laboratoire Ingenierie Circulation Transport (ENTPE / INRETS)

His research interests include traffic flow theory, experimental analysis of traffic flow behavior to improve the overall understanding and environmental assessment of transportation impacts. 